\section{FLEXIBLE RESTORATIONS TO SUIT YOUR NEEDS}

CEREC is the unique CAD/CAM system for individual all-ceramic restorations in a single appointment.

To meet dentists' individual needs Sirona has expanded their range to include two camera solutions and three different milling units. All components of the CEREC portfolio can be flexibly combined. Sirona has complied three configuration packages to make the decision making process even easier for you.

CEREC Classic is perfect if you're looking to focus on fully anatomical single tooth restorations. This economical entry level CEREC package includes CEREC AC with CEREC Bluecam, CEREC Software 4.2 and the CEREC MC milling machine.

CEREC Advanced is the system for dentists who want more. As well as fully anatomical single tooth restorations you can create perfectly fitting bridges, abutments and surgical guides. CEREC Advanced comprises CEREC AC with CEREC Omnicam, CEREC
Software 4.2 and CEREC MC X.

To complete your choice is CEREC Premium. Take advantage of the complete restoration and material diversity offered by CEREC and inLab software from Sirona. Produce chairside inlays, onlays, crowns, veneers and customised abutments to name just a few. In addition, you can mill or grind zirconium oxide bridges, bars, attachments and NPM restorations depending on material in your practice laboratory. CEREC Premium includes CEREC AC with CEREC Omnicam, CEREC Software 4.2 and CEREC MC XL premium package.

Call 08450715040 or email info@sironadental.co.uk.

\title{
AN INTRODUCTION TO DENTAL SLEEP MEDICINE
}

The British Society of Dental Sleep Medicine (BSDSM) is holding an introductory course for dentists and dental technicians called 'The Management of Snoring and Sleep Apnoea in Dental Practice'.

The course will give an overview of sleep disordered breathing; explain how oral appliances work and how to safely assess and monitor patients; give an overview of the medico-legal issues involved; introduce the patient overnight monitoring advice; evaluate appliances; and offer practical advice on introducing a dental sleep medicine service into your practice.

The course will take place on Saturday 18 October from 9.30 am until 5.30 pm in London and will include hands-on experience practising protrusive registration, fitting a selection of devices and group discussion.

Delegates will receive a comprehensive course manual, as well as the BSDSM screening protocol.

For further information visit www.dentalsleepmed. org.uk/shop.

\section{A HIGH RETURN ON YOUR INVESTMENT}

When you prescribe an Oralift appliance you can earn up to $£ 2,000$. Quick and easy to do, prescribing Oralift appliances offers a high return on your investment.

The Oralift device is designed to reduce and delay the signs of facial ageing (crow's feet, wrinkles, loss of skin tone, sagging jowls etc) without the need for surgery, needles or artificial fillers.

Oralift is a thermoplastic appliance which is easy to fit over the mandibular teeth similarly to a small mouthguard. It is worn for between 30 minutes and two hours, every third day for four months initially.

After this, two month top-ups twice a year is all that is needed. Requiring no exercise programme, the facial muscles are simply activated to create the rejuvenating effect by just wearing it in the mouth. In a matter of weeks, patients will start to notice Oralift's rejuvenating effects, which can become stronger and more pronounced over time.

To learn more about the Practice Building Benefits of Oralift attend one of Nick Mohindra's two-day hands-on courses. The $£ 650$ course fee includes 12 hours' verifiable CPD and a free set of Oralift appliances worth $£ 180$. The next course will be held in London on 5-6 September, with another course on 5-6 December. To book a place visit www.oralift.com or email info@oralift.com.

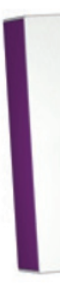

\section{HELPS STOP BLEEDING GUMS}

Corsodyl is set to relaunch its toothpaste range with a new hardhitting claim: 'Helps stop bleeding gums'. The Corsodyl toothpaste will be strengthened with a medical device classification from July onwards, meaning the new stronger medical claim will appear across packaging, TV and digital advertising and in-store POS.

The new engaging TV creative will feature Corsodyl toothpaste alongside its medicated mouthwash, further reinforcing the medical messaging across the range. Designed to generate awareness of Corsodyl toothpaste, the TV campaign will target both new and existing consumers, communicating the importance of acknowledging bleeding gums, and drive the message that Corsodyl toothpaste contains $70 \%$ specialised ingredients and can be used as part of a daily oral health regime.

Corsodyl Toothpaste is available in Original, Extra Fresh and Whitening at RRP €3.99.

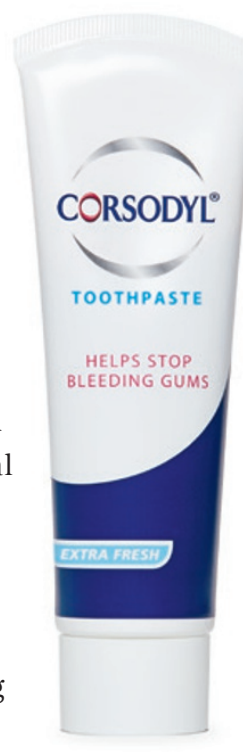

\section{GUTTA PERCHA TRIMMER}

QED's cordless Gutta Percha Trimmer is an extremely useful piece of equipment for GDPs and endodontic specialists.

Cordless for optimum flexibility and convenience, it is extremely lightweight, which helps minimise operator fatigue, and is extremely fast and easy to use. The kit comes complete with a handpiece, four interchangeable cutting tips, a handpiece holder, two rechargeable batteries and a battery charger.

Call 01733404999 or visit www.qedendo.co.uk. 